\title{
The Utility of Concentric Needle Single Fiber Electromyography in Diagnosing Myasthenia Gravis
}

\author{
Ahmed Sameer Nadeem*, Kawthar Salim AL-Salmi and Abdullah Mohammed AL-Salti \\ Neurology Department, Khoula Hospital-MOH. Muscat, Oman
}

Submission: March 11, 2021; Published: May 05, 2021

*Corresponding author: Ahmed Sameer Nadeem, Department of Neurology, Clinical neurophysiology unit, Khoula Hospital-MOH, Oman

Abstract

Introduction: Myasthenia Gravis (MG) is a chronic autoimmune neuromuscular disorder, characterized by varying degrees of fluctuating muscular weakness and fatigability. The diagnosis of MG can be difficult and, therefore, bed-side clinical testing, electrophysiological investigations; as well as serum antibodies tests are often used to establish the diagnosis.

Objective: The aim of this study was to define the diagnostic accuracy of concentric needle electrode single fiber electromyography (CNESFEMG) jitter test in MG patients and to determine its' diagnostic value by calculating the sensitivity, specificity, predictive values and accuracy of the test.

Methods: Retrospective analysis of 74 Omani MG patients' data was carried out to determine the diagnostic sensitivity, specificity and predictive values of CNE-SFEMG test; against a reference standard of MG by virtue of clinical features, antibody assay and response to treatment.

Receiver Operating Characteristic (ROC) curve was plotted and the area under the ROC curve (AUC) was also calculated to measure the accuracy of the test. A comparison of the frequency of CNE-SFEMG abnormality between ocular (OMG) and generalized (GMG) myasthenia gravis patients was done.

Results: Abnormal CNE-SFEMG results were detected in 79.1\%, 77\% and 82.4\% of all MG, GMG and OMG patients respectively. Based on the reference standard used, the sensitivity, specificity and predictive values were determined, and the test accuracy was measured in all MG, GMG and OMG included patients.

CNE-SFEMG had a sensitivity of $79.1 \%$ and specificity of $51.6 \%$ in all MG patients with $67.6 \%$ test accuracy. In GMG, CNE-SFEMG had a sensitivity of $76.9 \%$, specificity of $35.7 \%$ and $62.5 \%$ accuracy of the test; while CNE-SFEMG for OMG cases showed a sensitivity of $82.4 \%$ and specificity of $64.7 \%$ with $73.5 \%$ test accuracy.

The CNE-SFEMG abnormality was more frequent in the ocular than the generalized MG patients but with insignificant statistical difference.

Conclusion: Our findings indicate that CNE-SFEMG is a very useful and valid test for MG and our results were comparable, but lower than previously published values.

Significance: The sensitivities, specificity, predictive values and test accuracy of CNE-SFEMG jitter in the diagnosis of MG cases attending the neuromuscular unit at a tertiary care hospital in Muscat-Oman were documented.

Keywords: Myasthenia gravis (MG); Concentric needle electrode; Single fiber electromyography; EMG; SFEMG; Sensitivity; Specificity; Predictive values; Test accuracy

\section{Introduction}

Myasthenia Gravis (MG) is a chronic autoimmune neuromuscular disorder, characterized by varying degrees of fluctuating weakness and fatigability of the skeletal muscles without loss of reflexes or impairment of sensation or any other neurologic function [1]. It is a relatively common neurological disease resulting from an antibody-mediated neuromuscular transmission defect, where antibodies are directed against the muscle nicotinic acetylcholine receptors (AChR) or, sometimes, against other post junctional components such as the muscle specific kinase (MuSK) which indirectly decreases the AChR numbers [2,3]. The disease can occur at any age, however onset in the first decade is relatively rare $(10 \%)$ and it has two peaks for age of onset: between 20 -30 years in female and between 50-60 years in male [4]. The majority of patients present initially with 


\section{Open Access Journal of Neurology \& Neurosurgery}

ocular symptoms of fatigable ptosis and/or extra ocular muscle weakness causing diplopia (in up to $65 \%$ of patients) and resulting in ocular MG (OMG). Some progress to involve extraocular areas or present initially with increasing muscle fatigue, bulbar and/or proximal limbs' weakness causing generalized MG (GMG) [4-6]. Few patients may present with neuromuscular respiratory failure from the onset [4].

Despite the fact that the symptoms of MG are characteristic, they are common complaints which can be found in variety of medical and even psychological conditions; in addition, these symptoms are not specific to the condition. Hence, the diagnosis of MG can be difficult due to these facts, particularly in mild cases or when the physician is not familiar enough to suspect the condition. Moreover, it is realized that there is no single test that absolutely proves the diagnosis of MG and the combination of history, clinical examination, bed-side clinical testing (ice test and the Tensilon/Neostigmine test), electrophysiological investigations and serum anti-bodies tests often establishes the diagnosis beyond reasonable doubt [7]. In some cases all of these confirmatory tests are negative, although the clinical examination points to MG. In such cases, considered as possible MG or probable $\mathrm{MG}$, the patient would be monitored and followed up to check if the condition manifests itself and, sometimes, a diagnostic trial of treatment may be given [8]. Although the clinical information remains the 'gold or reference standard' used for the diagnosis of MG and for ruling out other disorders, diagnostic tests are also used and have been discussed extensively, in terms of reliability, sensitivity and specificity [5,8-12]. The usefulness of the bedside clinical tests has some limitations, e.g. the icepack test, although simple and quick, is only useful when there is eyelid drooping [13] and the Tensilon/Neostigmine test may not be easily interpretable when fatigability is not obvious, not completely reliable, less welltolerated and can only be done in hospital $[8,14]$. Generally, the presence of antibodies to Acetylcholine receptor (anti-AChR) and anti-MuSK antibodies are highly diagnostic and significant of MG, however, the absence of such antibodies does not exclude MG. Moreover, these are more often positive in GMG than OMG, and this may limit their usage in the latter common presentation $[5,8]$. Electrophysiological testing of the neuromuscular junction showing decrement on Repetitive Nerve Stimulation (RNS) usually confirms MG, but the test is frequently normal in patients with mild GMG or in purely OMG disease; furthermore, RNS has a lower sensitivity than Single Fiber Electromyography (SFEMG) in the diagnosis of MG $[9,14]$. On the other hand, the finding of increased jitter by SFEMG is the most sensitive electrophysiological test for abnormal neuromuscular junction transmission and it is abnormal in almost all patients with myasthenia gravis [11]. However, increase jitter on SFEMG does not prove myasthenia gravis and can be present in a number of other conditions that affect the nerve and muscle, which can also cause symptoms similar to that of MG, like in diabetes mellitus, neuropathy, myopathy, previous local trauma, as well as after surgery $[8,11,12]$.
SFEMG is found to be reliable and of high sensitivity for the diagnosis of MG [11]. The validity of the technique has been proven by studies including large number of MG patients, and a sensitivity of up to $99 \%$ in detecting a neuromuscular transmission defect in GMG has been reported [15-17] with the standard method, which uses a specialized electrode having an active recording core set at the side of a cannula [18]. These specialized electrodes are for single use (or reusable after sterilization) and expensive adding much cost especially in centers performing large number of examinations. In addition, for health and safety reasons, with the increasing concerns about possible transmission of prion disease by iatrogenic means after the isolation of prion proteins in the muscles of affected patients [19], several workers have modified the standard SFEMG technique by using a disposable Concentric Needle Electrode (CNE), with appropriate low-cut filters $[18,20,21]$. Normal values were established for this modified technique, its efficiency was confirmed in the diagnosis of $\mathrm{MG}$ and results showed that the modified technique, with voluntary activation, gives very similar normal values to the established SFEMG method, but the studies were done on a relatively smaller number of patients $[16,18,20-22]$ than those done with standard SFEMG. This study aims to determine the utility of concentric needle electrode single fiber electromyography (CNE-SFEMG) in the diagnosis of Myasthenia Gravis (MG) calculating the sensitivity and specificity of the test in Omani patients with a clinically definite disease.

\section{Subjects and Methods}

This cross-sectional retrospective study was conducted on diagnosed MG patients attending the Neuromuscular ClinicNeurology Department at Khoula Hospital, Muscat-Oman during the period from January 2016 to the end of December 2019, after obtaining ethical approval by the Institutional Research Ethics Committee (code No. PRO102020069).

Only definite cases of MG according to the following criteria [1] were included:

\section{A. Characteristic signs and symptoms}

\section{One or more of the following:}

1. Diplopia, ptosis, dysarthria, weakness in chewing, difficulty in swallowing, muscle weakness with preserved deep tendon reflexes, and, less commonly, weakness of neck extension and flexion, and weakness of trunk muscles

2. Increased weakness during exercise and repetitive use with at least partially restored strength after periods of rest

3. Dramatic improvement in strength following administration of anti-cholinesterase drug (edrophonium (Tensilón $®$ ) and/or Neostigmine) or a positive clinical response to treatment with pyridostigmine, corticosteroids or other immunomodulation therapy. 
4. Antibodies to Acetylcholine Receptors or anti-MUSK antibodies.

\section{B. Exclusions:}

1. Congenital myasthenic syndrome, progressive restricted myopathies, steroid and inflammatory myopathies, motor neuron disease, Multiple sclerosis, variants of Guillain-Barré syndrome (e.g., Miller-Fisher syndrome), Eaton-Lambert syndrome, Stroke.

2. Prior strabismus surgery as well as any other cause of paralytic strabismus.

3. Organophosphate toxicity, botulism, black widow spider venom.

4. Medications: neuromuscular blocking agents, aminoglycosides, penicillamine, antimalarial drugs, colistin, streptomycin, polymyxin B, tetracycline

\section{Diabetes, Hypokalemia; hypophosphatemia}

SFEMG was not considered a criterion in making the MG diagnosis and the mentioned confounders are minimized by the selection criterion.

\section{Procedure}

All MG Patients' electronic files were reviewed for extracting epidemiological and clinical data, as well as for obtaining the investigations and various tests' results. Each record was evaluated regarding details of history and clinical examination, including age of patients at presentation, presence of ocular symptoms, easy fatigability / generalized weakness, bulbar involvement, proximal upper/lower limb weakness. Details of different diagnostic modalities utilized for confirmation of MG were analyzed, including Neostigmine test, concentric needle SFEMG, Repetitive Nerve stimulation test and anti-AChR and AntMuSK Antibody tests. Response to various treatment used was also evaluated from follow up visits. All patients were clinically classified using the the Myasthenia Gravis Foundation of America (MGFA) Clinical Classification [23]; but due to the small cohort number, we have divided our patients into two groups:

I. OMG, was considered in included patients with variable fatigable ptosis and/or having variable ophthalmoplegia. Exclusion criteria included previous strabismus surgery and any other reason of paralytic strabismus.

II. GMG, was defined clinically in the included patients as OMG patients presenting with or progressing onto having motor weakness, causing one of the following: dysphagia, dysarthria, dyspnea, or remote motor weakness, involving the neck or the extremities [24].

All SFEMG assessments were performed according to the International Federation of Clinical Neurophysiology (IFCN)endorsed Guideline for measurement of neuromuscular jitter with concentric needle electrodes (CNE - CN jitter) [17]. Dantec
Keypoint (Natus Medical, Inc., San Carlos, California) EMG machine was used for measurements of SFEMG parameters with voluntary muscle activation technique. Recording was made using a disposable concentric needle electrode with a diameter of 0.3 $\mathrm{mm}$ and recording area of $0.02 \mathrm{~mm}^{2}$ (Natus neurology-Dantec ${ }^{\mathrm{TM}}$ DNC disposable needle electrodes, 9013S0012; Gort, co. Galway, Ireland). The test was considered as abnormal if the mean (or median) jitter exceeds the upper limit per age for the muscle examined (Extensor Digitorum Communis EDC or Frontalis muscle) or if more than $10 \%$ of pairs (at least 2 of 20 recorded responses) have increased jitter or blocking [20,21].

\section{Statistical analysis}

For statistical purpose, the reference standard used to designate the presence of MG in a patient was the presence of clinical features of OMG or GMG in association with one or both of the following clinical investigations:

1. positive anti-AChR or Anti-MuSK antibodies,

2. positive clinical response to treatment with, one or a combination of, pyridostigmine, corticosteroids or other immunomodulation therapy. This was to insure as accurately as possible the diagnosis of MG, including the sero-negative type [24] and to form a reference standard against which CNESFEMG sensitivity, specificity and predictive values (PPV-positive predictive value, NPV-negative predictive value) are calculated. Those patients presenting with clinical complaints as stated, but without antibody positivity or response to treatment, were considered as non-MG patients.

To describes the relationship between the true positive rate (sensitivity) of a test versus its false positive rate (1-specificity) for different cut-off points associated with a diagnostic test graphically and to display the trade-off relationship between sensitivity and specificity for all possible thresholds, the Receiver Operating Characteristic (ROC) curve was plotted and the area under the ROC curve (AUC) was also calculated, using online web-base program for both [25], to measure the accuracy of the diagnostic test. Chi-square statistic between OMG and GMG for CNE-SFEMG abnormal results was performed.

\section{Results}

A total of 74 Omani patients were included in this study, including 54 females and 20 males with female: male ratio of 2.7:1. The mean age for all patients was $34.81 . \pm 11.8$ years. In male patients, the mean age was $37.5 \pm 14.48$ (range 13-75) years; while female patients had a mean age was $33.81 \pm 10.75$ (range 17-69) years. According to the clinical findings, 40 patients (54\%) had GMG and 34 (46\%) where of OMG. Based on the reference standard used, out of 74 MG patients, 43 patients were classified as MG patients and 31 were non-MG patients. 34/43 (79.1\%) MG patients had positive SFEMG and only 9/43 (20.9\%) had negative SFEMG. Of the non-MG patients, 15/31 (48.4\%) had 


\section{Open Access Journal of Neurology \& Neurosurgery}

positive SFEMG and 16/31 (51.6\%) had negative SFEMG. For all MG patients, SFEMG had a sensitivity of $79.1 \%$ (PPV=69.0\%) and specificity of only $51.6 \%$ (NPV $=64 \%$ ). The area under the ROC curve for the SFEMG was (0.653) with $67.6 \%$ accuracy of the test (Figure 1). When GMG patients were considered separately, 26 patients were classified as MG patients and 14 were non-MG patients. 20/26 (77\%) MG patients had positive SFEMG and only $6 / 26$ (23\%) had negative SFEMG. Of the non-MG patients, 9/14 (64\%) had positive SFEMG. SFEMG for GMG had a sensitivity of $76.9 \%$ (PPV=69\%), specificity of $35.7 \%(\mathrm{NPV}=45.5 \%)$ and the area under the ROC curve for the GMG SFEMG was (0.563) with $62.5 \%$ accuracy of the test (Figure 2). Similarly, when OMG is considered separately, 17 patients were classified as MG patients and 17 were non-MG patients. 14/17 (82.4\%) OMG patients had positive SFEMG and only 3.0/17 (17.6 \%) had negative SFEMG. Of the non-MG patients, $6 / 17$ (35.3\%) had positive SFEMG and $11 / 17(64.7 \%)$ had negative SFEMG. SFEMG for OMG showed a sensitivity of $82.4 \%$ (PPV $=70 \%$ ) and specificity of $64.7 \%$ (NPV $=78.6 \%$ ). The area under the ROC curve for the OMG SFEMG was (0.735) with $73.5 \%$ accuracy of the test (Figures $3 \& 4$ ). Although the abnormal CNE-SFEMG results are more frequent in the OMG than the GMG patients, the chi-square statistic showed no significant differences $(\mathrm{P}>0.05)$ Table 1.

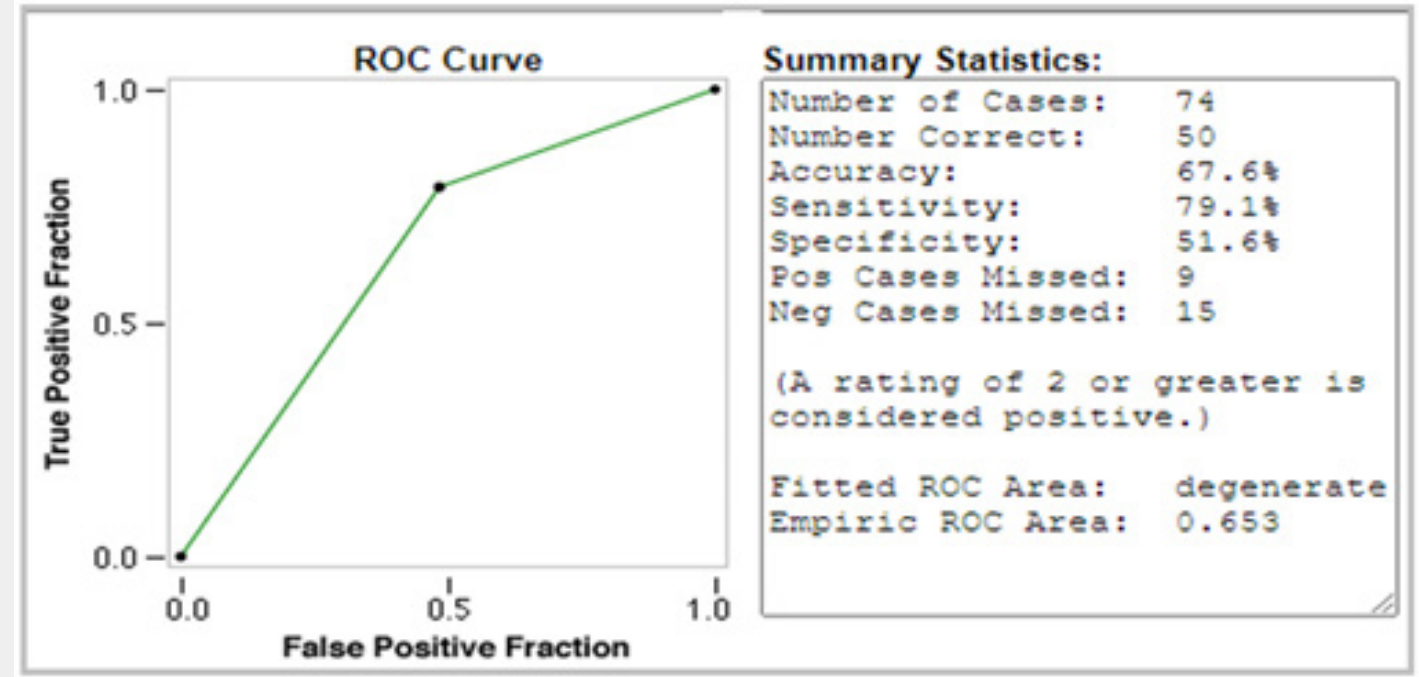

Figure 1: Receiver operating characteristic (ROC) curve space of the CNE-SFEMG using reference standard for MG diagnosis for all patients with MG.

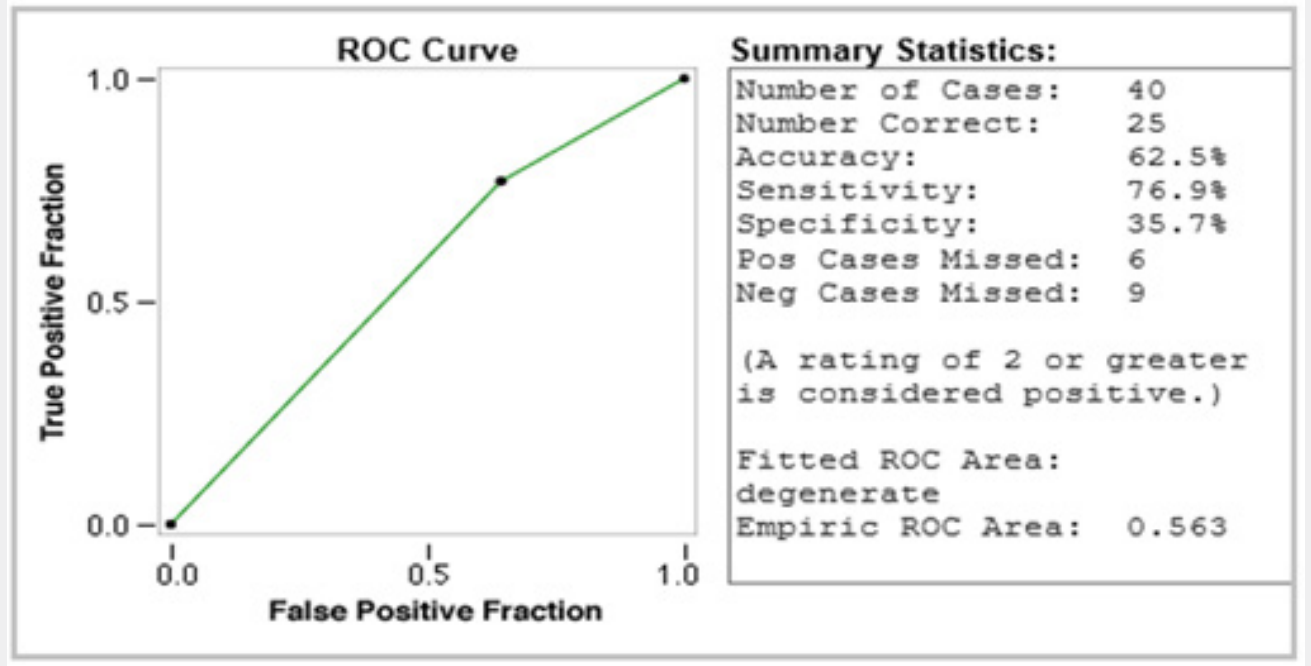

Figure 2: Receiver operating characteristic (ROC) curve space of the CNE-SFEMG using reference standard for MG diagnosis for patients with GMG. 


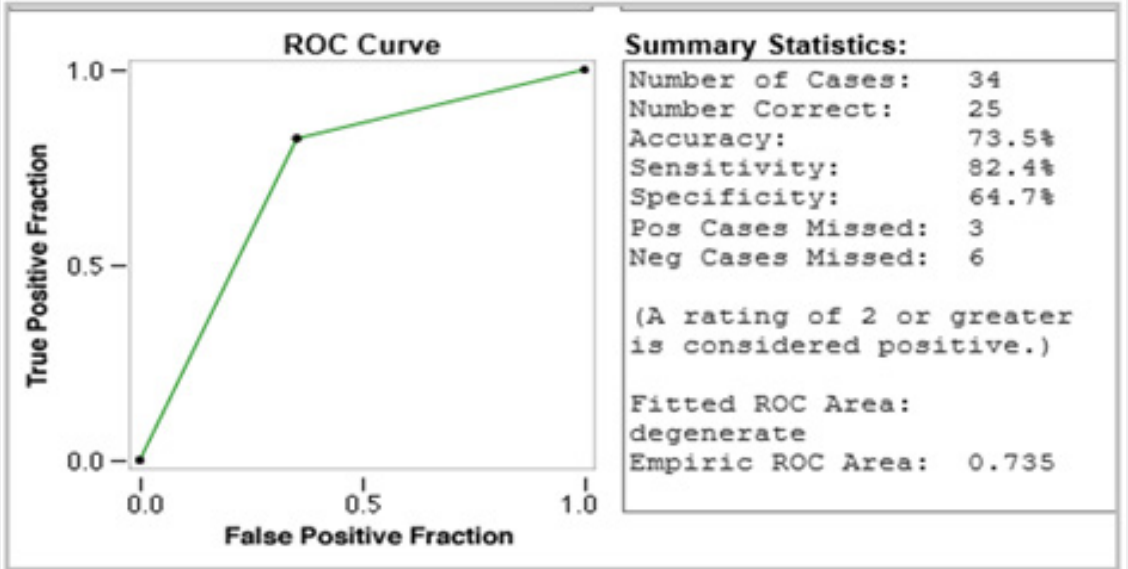

Figure 3: Receiver operating characteristic (ROC) curve space of the CNE-SFEMG using reference standard for MG diagnosis for patients with OMG.

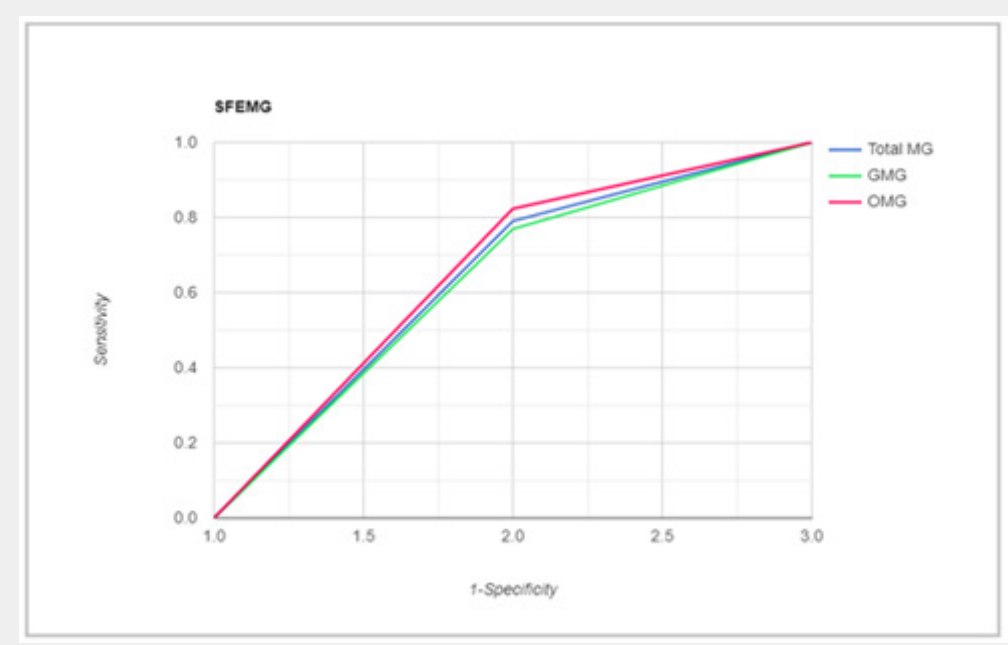

Figure 4: Comparison for Receiver operating characteristic (ROC) curves space of the CNE-SFEMG using reference standard for MG diagnosis for all MG patients, GMG and OMG patients.

Table 1: chi-square statistic between OMG and GMG for CNE-SFEMG results.

\begin{tabular}{|c|c|c|c|}
\hline & SFEMG+ & SFEMG- & Marginal Row Totals \\
\hline OMG & $14(13.44)[0.02]$ & $3(3.56)[0.09]$ & 17 \\
\hline GMG & $20(20.56)[0.02]$ & $6(5.44)[0.06]$ & 26 \\
\hline Marginal Column Totals & 34 & 9 & $43(\mathrm{Grand}$ Total) \\
\hline
\end{tabular}

The chi-square statistic is 0.1831 . The $p$-value is .668701 . Not significant at $p<.05$.

The chi-square statistic with Yates correction is 0.002 . The $p$-value is .964445 . Not significant at $p<.05$.

\section{Discussion}

MG is the most common acquired disorder of neuromuscular transmission [26] and although it is a potentially serious disorder, MG is treatable, and the vast majority of patients improve with therapy over time. Some can often go into remission or minimal manifestation status [23]. MG can be difficult to diagnose as symptoms can mimic variety of disorders; in addition to the fact that MG symptoms may be vague, fluctuate or only affect certain muscles in mild or early cases. Thus, it is not unusual for a diagnosis of MG to be delayed or even misdiagnosed leading to unnecessary and potentially harmful therapeutic interventions, as well as to treatment delay and, hence, to increased morbidity [27]. 


\section{Open Access Journal of Neurology \& Neurosurgery}

Therefore, it is critically important to have an accurate and early diagnosis. Since there is no single test which could absolutely proves the diagnosis of MG and a combination of tests are used, with history and clinical examination, [7] it is important to evaluate the usefulness of these tests. Determining the sensitivity and specificity of an investigation is a powerful gold standard required to assign patients into a disease group.

Few papers have reported the sensitivity of CNE-SFEMG in MG [28-34], and a small number of them have also addressed their specificity $[24,28,29,33]$. Our study's findings reaffirm the high sensitivity of the CNE-SFEMG test in the diagnosis of MG. Abnormal CNE-SFEMG results were detected in $79.1 \%, 77 \%$ and $82.4 \%$ of all MG, GMG and OMG patients respectively. Based on the

Table 2: Reported sensitivity of CNE jitter studies in myasthenia gravis.

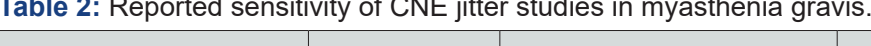

\begin{tabular}{|c|c|c|c|c|c|c|c|}
\hline Reference & $\begin{array}{l}\text { Population } \\
\text { Studied }\end{array}$ & Diagnostic Criteria & Muscle & Activation & MG Class & Sensitivity & Specificity \\
\hline $\begin{array}{c}\text { Benatar et al. } 2006 \\
\text { [28] }\end{array}$ & $\begin{array}{l}21 \mathrm{MG} \text { vs } \\
30 \text { non-MG }\end{array}$ & $\begin{array}{l}\text { AChR antibody or re- } \\
\text { sponse to treatment }\end{array}$ & Front & Vol & $\begin{array}{c}\text { OMG } \\
\text { GMG } \\
\text { All }\end{array}$ & $\begin{array}{l}62 \% \\
75 \% \\
67 \%\end{array}$ & $96 \%$ \\
\hline $\begin{array}{c}\text { Sarrigiannis et al. } 2006 \\
\text { [29] }\end{array}$ & $\begin{array}{c}\text { Normal } 56 \\
\text { Vs } \\
\text { MG } 20\end{array}$ & AChR antibody & $\begin{array}{c}00 \\
(N=30) \\
E D \\
(N=53)\end{array}$ & Vol & All & $96 \%$ & $100 \%$ \\
\hline $\begin{array}{l}\text { Kouyoumdjian et al. } \\
2011[30]\end{array}$ & $\begin{array}{c}M G \\
N=20\end{array}$ & $\begin{array}{l}\text { AChR antibody, or } \\
\text { RNS plus response to } \\
\text { treatment }\end{array}$ & $\begin{array}{l}\text { ED or } \\
\text { Front }\end{array}$ & Stim & $\begin{array}{l}\text { OMG } \\
\text { GMG } \\
\text { All }\end{array}$ & $\begin{array}{c}75 \% \\
94 \% \\
\text { ED-85\% } \\
\text { Front-85\% } \\
\text { Either-90\% }\end{array}$ & ND \\
\hline $\begin{array}{l}\text { Witoonpanich et al., } \\
2011 \text { [31] }\end{array}$ & $\begin{array}{c}\text { MG } \\
N=112\end{array}$ & $\begin{array}{l}\text { Clinical, plus response } \\
\text { to ch'ase inhibitors and } \\
\text { treatment }\end{array}$ & ED or 00 & Vol & $\begin{array}{c}\text { OMG } \\
(\mathrm{N}=42) \\
\text { GMG } \\
(\mathrm{N}=70)\end{array}$ & $\begin{array}{l}93 \% \\
99 \%\end{array}$ & ND \\
\hline $\begin{array}{l}\text { Kouyoumdjian and } \\
\text { Stålberg. } 2013 \text { [32] }\end{array}$ & $\begin{array}{c}\mathrm{MG} \\
\mathrm{N}=42\end{array}$ & $\begin{array}{l}\text { AChR antibody, or clinical } \\
\text { plus } \\
\text { response to ch'ase inhibi- } \\
\text { tors or RNS }\end{array}$ & $\begin{array}{l}\text { ED or } \\
\text { Front }\end{array}$ & Stim & $\begin{array}{l}\text { OMG } \\
\text { GMG }\end{array}$ & $\begin{array}{c}\text { ED- } 67 \% \\
\text { Front- } 83 \% \\
\text { Either- } 90 \% \\
\text { ED or Front } 89 \%\end{array}$ & ND \\
\hline $\begin{array}{l}\text { Machado et al..2017 } \\
\text { [33] }\end{array}$ & $\begin{array}{c}33 \text { MG vs } 20 \\
\text { normal }\end{array}$ & $\begin{array}{l}\text { AChR antibody, or } \\
\text { clinical plus response to } \\
\text { ch'ase inhibitors or RNS }\end{array}$ & 00 & Vol & $\begin{array}{l}\mathrm{OMG} \\
\mathrm{GMG}\end{array}$ & $\begin{array}{l}92.3 \% \\
100 \%\end{array}$ & $96 \%$ \\
\hline Lo et al. 2017 [24] & $\begin{array}{c}M G \\
N=127\end{array}$ & $\begin{array}{l}2 \text { reference standards of } \\
\text { MG by virtue of clinical } \\
\text { features, antibody assay } \\
\text { and response to treat- } \\
\text { ment }\end{array}$ & 00 & Stim & $\begin{array}{c}\text { Ref.1: } \\
\text { All } \\
\text { OMG } \\
\text { GMG } \\
\text { Ref.2: } \\
\text { All } \\
\text { OMG } \\
\text { GMG }\end{array}$ & $\begin{array}{c}97.3 \% \\
100 \% \\
83.3 \% \\
\\
93.8 \% \\
100 \% \\
75 \%\end{array}$ & $\begin{array}{l}21 \% \\
21.8 \% \\
\text { ND }\end{array}$ \\
\hline
\end{tabular}

reference standard used, CNE-SFEMG had a sensitivity of $79.1 \%$ and specificity of $51.6 \%$ in all MG patients. In GMG a sensitivity of $76.9 \%$ and specificity of $35.7 \%$ is calculated for the test; while CNE-SFEMG for OMG cases showed a sensitivity of $82.4 \%$ and specificity of $64.7 \%$. These results are comparable to the previous studies in the calculated sensitivity; however, three studies report higher $(96-100 \%)$ specificity $[28,29,33]$ and one study reported $17.2-17.9 \%$ specificity in a large Asian cohort [24]. Receivers Operating Characteristic (ROC) curves were also constructed for our patients' results, to define maximal sensitivity and specificity of the technique and to demonstrate the diagnostic accuracy of CNE-SFEMG, showing $67.6 \%, 62.5 \%$ and $73.5 \%$ accuracy for all MG, GMG and OMG respectively. Higher ROC curves' values are reported in other studies [24,33]. 


\section{Open Access Journal of Neurology \& Neurosurgery}

\begin{tabular}{|c|c|c|c|c|c|c|c|}
\hline Sirin et al. 2018 [34] & $\begin{array}{c}\text { MG } \\
\text { (untreated) } \\
\mathrm{N}=30\end{array}$ & $\begin{array}{l}\text { AChR or MuSK antibody, } \\
\text { or clinical plus response } \\
\text { to ch'ase inhibitors }\end{array}$ & $\begin{array}{l}\text { ED } \\
\text { Front }\end{array}$ & Stim & $\begin{array}{l}\text { OMG } \\
\text { GMG }\end{array}$ & $\begin{array}{l}\text { ED or Front- } 93 \% \\
\text { ED or Front- } 100 \%\end{array}$ & ND \\
\hline This Study & $\begin{array}{c}\mathrm{MG} \\
\mathrm{N}=74\end{array}$ & $\begin{array}{l}\text { Clinical, plus AChR / } \\
\text { MuSK antibody or } \\
\text { response to ch'ase inhibi- } \\
\text { tors and treatment }\end{array}$ & $\begin{array}{l}\text { ED } \\
\text { Front }\end{array}$ & Vol & $\begin{array}{l}\text { GMG } \\
\text { All }\end{array}$ & $\begin{array}{l}\text { ED or Front- } 82.4 \% \\
\text { ED or Front }-76.9 \% \\
\text { ED or Front }-79.1 \%\end{array}$ & $\begin{array}{r}64 \% \\
35.7 \% \\
51.6 \%\end{array}$ \\
\hline
\end{tabular}

AChR - acetylcholine receptor; ch'ase - cholinesterase; Clinical - fluctuating weakness by history and examination; ED - extensor digitorum muscle; Front - frontalis muscle; GMG - generalized myasthenia gravis; MuSK - muscle specific tyrosine kinase; ND - not done; OMG - ocular myasthenia gravis; OO- orbicularis oculi; stim - stimulation; vol - voluntary.

For most previously published studies, CNE-SFEMG has a higher sensitivity in GMG and a relatively lower sensitivity in OMG [28-34]. However, our series showed a higher sensitivity in OMG than in GMG, a similar finding was reported by Lo et al. 2017 [24], and the frequency of CNE-SFEMG abnormality was greater in the ocular than the generalized MG patients in our study but without significant statistical difference Table 2 summarizes the reported sensitivity of CNE jitter studies in myasthenia gravis including this study. This variability in the reported CNE-SFEMG sensitivity and specificity results' percentages can be explained, in part, by methodological differences $[8,24]$. The lack of a reference gold standard is also an important factor for these differences. Measuring the sensitivity of a diagnostic test requires that it should be compared with a gold standard diagnostic criterion that is independent of the test in question. We selected to use a clinical diagnosis of myasthenia gravis made by an expert in neuromuscular diseases by combination of clinical findings and raised AChR/ MuSK antibody titers (themselves reported to be highly specific for myasthenia gravis, although sero-negative MG do exists) [35], OR evident clinical response to treatment. The different choice of specific muscles and the number of muscles tested are important methodological factors that can be responsible for the variability between the different studies. Most studies report the results of jitter testing in the same one or two muscles in all patients, even though no one muscle, or combination of muscles is more likely to be abnormal in MG patients [24].

The relative proportions of MG clinical phenotypes included in the different studies may account for these differences too [8], as cases with GMG can be often diagnosed efficiently (with positive antibodies, RNS and bedside clinical testing, in addition to their clinical features), without going to the invasive SFEMG testing, affecting their proportion in the selected subjects' samples. Furthermore, the sample size, incorporation bias, non-blinding, inclusion of non-representative populations and different ethnic groups can be additional factors in such reported differences [24]. This study, however, has some limitations that need to be reported.
The modest number of patients included is the first limitation. Secondly, the considerable number of patients with a negative diagnosis of MG (according to the reference standard used) whom might have positive anti-MuSK antibodies, as anti-MuSK testing was not performed to all of our patients (only in 42 patients out of the 74 included) and finally, the retrospective nature of this study which implies a few inherent limitations, like, a homogenous testing of all patients. To our knowledge, this is the first study of this nature to date in Oman, and our findings can have relevant implications for the management and future research of MG.

\section{Conclusion}

Our findings indicate that, in Omani cohort of definite MG patients, CNE-SFEMG is a very useful and valid test. The sensitivity, specificity and predictive values were determined, and the test accuracy was measured in All MG, GMG and OMG included patients; in addition, a comparison between the frequency of test abnormality and clinical phenotypes was demonstrated.

Our results were comparable, but lower than previously published values, and the frequency of CNE-SFEMG abnormality was greater in the ocular than in the generalized MG patients' group but without significant statistical difference. The electrophysiological CNE-SFEMG test is affordable and less technically demanding approach for diagnosing MG, despite the reported poor specificity, and our results could provide physicians with more clear reliability using concentric needle electrode SFEMG for the diagnosis of MG. Further studies with larger population and from multiple centers are needed to be carried out to achieve more representative results.

\section{Declaration}

The authors declare that neither part nor all of the material is contained within any presentation made at any national or international meeting. 


\section{Ethical Publication Statement}

We confirm that we have read the Journal's position on issues involved in ethical publication and affirm that this report is consistent with those guidelines.

\section{Disclosure of Conflicts of Interest}

None of the authors has any conflict of interest to disclose and none of the authors had received any financial income/ payment from commercial sources, the interests of which are relevant to this research activity.

\section{Ethical Committee approval}

This study was approved by the relevant research ethics committee - The centre of Studies and Research, Directorate General of Planning and Studies-MOH, Oman (approval code No. PR0102020069).

\section{Significance}

Sensitivity, specificity, predictive values and test accuracy of the Concentric Needle Single Fiber Electromyography calculated on cases attending the neuromuscular unit at a tertiary care hospital, Oman.

\section{References}

1. Engel AG (1994) Myasthenic syndromes. In: Engel AG, Franzini Armstrong C, (eds). Myology. $2^{\text {nd }}$ Edition: McGraw Hill, New York, USA, pp. 1798-835.

2. De Baets M, Stassen MH (2002) The role of antibodies in myasthenia gravis. J Neurol Sci 202(1-2): 5-11.

3. Chan KH, Lachance DH, Harper DM, Lennon VA (2007) Frequency of seronegativity in adult-acquired generalized myasthenia gravis. Muscle Nerve 36(5): 651-658.

4. Kim WH, Kim JH, Kim EK, Yun SP, Kim KK, et al. (2010) Myasthenia gravis presenting as isolated respiratory failure: A case report. Korean J Intern Med 25 (1): 101-104.

5. Mori S, Shigemoto K (2013) Mechanisms associated with the pathogenicity of antibodies against muscle-specific kinase in myasthenia gravis, Autoimmun Rev 12(9): 912-917.

6. Kupersmith MJ (2009) Ocular myasthenia gravis: treatment successes and failures in patients with long term follow up. J Neurol 256 (8): 1314-1320.

7. Caliandro P, Evoli A, Stalberg E, Granata G, Tonali P, et al. (2009) The difficulty in confirming clinical diagnosis of myasthenia gravis in a seronegative patient: a possible neurophysiological approach. Neuromuscul Disord 19 (12): 825-827.

8. Benatar M (2006) A systematic review of diagnostic studies in myasthenia gravis. Neuromuscul. Disord 16(7): 459-467.

9. Lo YL, Dan YF, Leoh TH, Tan YE, Nurjannah S, et al. (2004) Effect of exercise on repetitive nerve stimulation studies: new appraisal of an old technique. J Clin Neurophysiol 21(2): 110-113.

10. Oh SJ, Kim DE, Kuruoglu R, Bradley RJ, Dwyer D (1992) Diagnostic sensitivity of the laboratory tests in myasthenia gravis. Muscle Nerve 15(6): 720-724.
11. Padua L, Caliandro P, Di Iasi G, Pazzaglia C, Ciaraffa F, et al. (2014) Reliability of SFEMG in diagnosing myasthenia gravis: sensitivity and specificity calculated on 100 prospective cases. Clin Neurophysiol 125(6): 1270-1273.

12. (2001) AAEM Quality Assurance Committee. American Association of Electrodiagnostic Medicine. Literature review of the usefulness of repetitive nerve stimulation and single fiber EMG in the electrodiagnostic evaluation of patients with suspected myasthenia gravis or Lambert-Eaton myasthenic syndrome. Muscle Nerve24: 1239.

13. Golnik KC, Pena R, Lee AG, Eggenberger ER (1999) An ice test for the diagnosis of myasthenia gravis, Ophthalmology 106(7): 1282-1286.

14. Lo YL, Dan YF, Leoh TH, Tan YE, Ratnagopal P (2003) Decrement in area of muscle responses to repetitive nerve stimulation. Muscle Nerve 27(4): 494-496.

15. Sanders DB (2002) Clinical impact of single-fiber electromyography. Muscle Nerve Suppl 11: S15-20.

16. Bromberg MB, Scott DM (1994) Single fiber EMG reference values: reformatted in tabular form. AD HOC Committee of the AAEM Single Fiber Special Interest Group. Muscle Nerve 17(7): 820-821.

17. Sanders DB, Arimura K, Cui L, Ertaș M, Farrugia ME, et al. (2019) Guidelines for single fiber EMG. Clin Neurophysiol 130(8): 1417-1439.

18. King JC, Dumitru D, Nandedkar S (1997) Concentric and single fiber electrode spatial recording characteristics. Muscle Nerve 20(12): 1525-1533.

19. Glatzel M, Abela E, Maissen M, Aguzzi A (2003) Extraneural pathologic prion protein in sporadic Creutzfeldt-Jakob disease. N Engl J Med 349(19):1812-1820.

20. Kouyoumdjian JA, Stålberg, EV (2007) Concentric needle single fiber electromyography: normative jitter values on voluntary activated Extensor Digitorum Communis. Arquivos de Neuro-Psiquiatria 65 (2b): 446-449.

21. Stålberg E, Sanders DB, Ali S, Cooray G, Leonardis L, et al. (2016) Reference values for jitter recorded by concentric needle electrodes in healthy controls: A multicenter study. Muscle Nerve 53(3): 351-362.

22. Ertas M, Baslo MB, Yildiz N, Yazici J, Oge AE (2000) Concentric needle electrode for neuromuscular jitter analysis. Muscle Nerve 23(5): 715719.

23. Jaretzki A $3^{\text {rd }}$, Barohn RJ, Ernstoff RM, Kaminski HJ, Keesey JC, et al. (2000) Myasthenia gravis: recommendations for clinical research standards. Task Force of the Medical Scientific Advisory Board of the Myasthenia Gravis Foundation of America. Neurology 55(1): 16-23.

24. Lo YL, Najjar RP, Teo KY, Tow SL, Loo JL, et al. (2017) A reappraisal of diagnostic tests for myasthenia gravis in a large Asian cohort. J Neurol Sci 376:153-158.

25. Eng J (2014) ROC analysis: web-based calculator for ROC curves. Baltimore: Johns Hopkins University.

26. Deenen JC, Horlings CG, Verschuuren JJ, Verbeek AL, van Engelen BG (2015) The epidemiology of neuromuscular disorders: a comprehensive overview of the literature. J Neuromuscul Dis 2(1): 73-85.

27. Al-Asmi A, Nandhagopal R, Jacob PC, Gujjar A (2012) Misdiagnosis of Myasthenia Gravis and Subsequent Clinical Implication: A case report and review of literature. Sultan Qaboos Univ Med J 12(1): 103-108.

28. Benatar M, Hammad M, Doss-Riney H (2006) Concentric-needle single-fiber electromyography for the diagnosis of myasthenia gravis. Muscle Nerve 34(2): 163-168. 
29. Sarrigiannis PG, Kennett RP, Read S, Farrugia ME (2006) Single-fiber EMG with a concentric needle electrode: validation in myasthenia gravis. Muscle Nerve 33(1): 61-65.

30. Kouyoumdjian JA, da Silva Fanani AC, Stålberg EV (2011) Concentric needle jitter on stimulated Frontalis and Extensor Digitorum in 20 myasthenia gravis patients. Muscle Nerve 44(6): 912-918.

31. Witoonpanich R, Dejthevaporn C, Sriphrapradang A, Pulkes T (2011) Electrophysiological and immunological study in myasthenia gravis: diagnostic sensitivity and correlation. Clin Neurophysiol 122(9) 1873-1877.

32. Kouyoumdjian JA, Stålberg EV (2013) Concentric needle jitter on voluntary activated frontalis in 20 healthy subjects. Muscle Nerve $47(3): 440-2$.

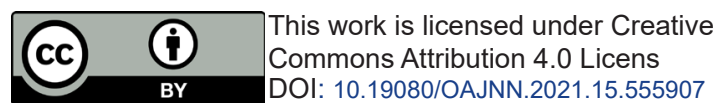

33. Machado FCN, Kouyoumdjian JA, Marchiori PE (2017) Diagnostic accuracy of concentric needle jitter in myasthenia: prospective study. Muscle Nerve 55(2): 190-194.

34. Sirin NG, Kocasoy Orhan E, Durmus H, Deymeer F, Baslo MB (2018) Repetitive nerve stimulation and jitter measurement with disposable concentric needle electrode in newly diagnosed myasthenia gravis patients. Neurophysiol Clin 48(5): 261-267.

35. Vincent A, Newsom-Davis J (1985) Acetylcholine receptor antibody as a diagnostic test for myasthenia gravis: results in 153 validated cases and 2967 diagnostic assays. J Neurol Neurosurg Psychiatry 48(12): 1246-1252.

\section{Your next submission with Juniper Publishers will reach you the below assets}

- Quality Editorial service

- Swift Peer Review

- Reprints availability

- E-prints Service

- Manuscript Podcast for convenient understanding

- Global attainment for your research

- Manuscript accessibility in different formats

( Pdf, E-pub, Full Text, Audio)

- Unceasing customer service

Track the below URL for one-step submission

https://juniperpublishers.com/online-submission.php 